\title{
Chirality Characterization of Dispersed Single Wall Carbon Nanotubes
}

Min Namkung ${ }^{1}$, Phillip A. Williams ${ }^{2}$, Candis D. Mayweather ${ }^{3}$, Buzz Wincheski ${ }^{1}$, Cheol Park ${ }^{4}$, and Juock S. Namkung

${ }^{1}$ NASA Langley Research Center, Hampton, VA 23681

${ }^{2}$ National Research Council, NASA Langley Research Center, Hampton, VA 23681

${ }^{3}$ Spelman College, Atlanta, Georgia 30314

${ }^{4}$ National Institute of Aerospace, Hampton, VA 23666

${ }^{5}$ Naval Air Warfare Center, Patuxent River, MD 20670

\begin{abstract}
Raman scattering and optical absorption spectroscopy are used for the chirality characterization of HiPco single wall carbon nanotubes (SWNTs) dispersed in aqueous solution with the surfactant sodium dodecylbenzene sulfonate. Radial breathing mode (RBM) Raman peaks for semiconducting and metallic SWNTs are identified by directly comparing the Raman spectra with the Kataura plot. The SWNT diameters are calculated from these resonant peak positions. Next, a list of (n, m) pairs, yielding the SWNT diameters within a few percent of that obtained from each resonant peak position, is established. The interband transition energies for the list of SWNT $(n, m)$ pairs are calculated based on the tight binding energy expression for each list of the $(n, m)$ pairs, and the pairs yielding the closest values to the corresponding experimental optical absorption peaks are selected. The results reveal that $(1,11),(4,11)$, and $(0,11)$ as the most probable chiralities of the semiconducting nanotubes. The results also reveal that $(4,16)$, $(6,12)$ and $(8,8)$ are the most probable chiralities for the metallic nanotubes. Directly relating the Raman scattering data to the optical absorption spectra, the present method is considered the simplest technique currently available. Another advantage of this technique is the use of the $E_{11}^{S}$ peaks in the optical absorption spectrum in the analysis to enhance the accuracy in the results.
\end{abstract}

\section{INTRODUCTION}

The physical properties of a single wall carbon nanotube (SWNT) depend on its chirality, which is determined by two integer coefficients, normally denoted by $(n, m)$ [1]. The spectroscopy methods of fluorescence, Raman scattering and optical absorption have been the mainstream tools for chirality characterization [2-4], and each method has unique capabilities. Fluorescence spectroscopy is a novel technique providing information on the chirality of SWNTs but its applicability is limited to semiconducting SWNTs. The Raman scattering technique directly provides the distribution of SWNT diameters which are closely related to the chirality as the diameter of an individual SWNT is determined by its (n, m). Optical absorption spectroscopy provides the interband transition energies, which are also closely related to the chirality distribution since the interband transition energies of a SWNT are an explicit function of its (n, $\mathrm{m})$ values. Unfortunately, both the SWNT diameter and interband transition energy are multivalued functions of (n, $m$ ). Hence, it is logical to combine the results of the Raman and optical absorption spectra of a sample for a unique determination of the chirality distribution. However, the wavelength of the excitation radiation for the optical absorption method varies continuously 
within a range, whereas the monochromatic laser beams used for the Raman spectroscopy possibly leave unmatched spectral regions.

Recently, two research groups in the US and Europe have employed tunable laser sources and obtained Raman spectra with the incident laser wavelength varying in a range $[3,5,6]$. This technique provides not only the Raman spectra but also the optical absorption spectra from a batch of SWNTs at the same time. This elegant technique ensures the one-to-one corresponddence between specific resonant Raman peaks and optical absorption peaks enabling a complete incorporation of two spectra in a given range. Despite such a clear advantage, the experimental procedure of this scanned Raman method with tunable lasers can be complex such that its application may be limited to research purposes only. In this paper, we present a simpler procedure that is capable of characterizing the chirality distribution of a batch of SWNTs. The method is based on the direct combination of the Raman and optical absorption spectra. In particular, we demonstrate that a near complete comparison between the two types of spectra is accomplished with limited Raman data.

\section{EXPERIMENTAL DETAILS}

Purified SWNTs synthesized through the HiPco process [7] are obtained from Carbon Nanotechnologies, Inc. Following procedures similar to those of Islam et al. [8] and Paredes et al. [9], the SWNT material is dispersed in an aqueous solution of the surfactant sodium dodecyl benzene sulfonate (NaDDBS). Briefly, the SWNT material as dispersed in the surfactant solution is subjected to ultrasonic agitation and centrifugation followed by decanting of the supernatant. This process suspends the SWNTs in the solution and exfoliates the larger bundles of SWNTs into smaller bundles and individual nanotubes for more accurate quantitative analysis of the SWNT chirality distribution.

Optical absorption measurements of the resulting supernatant are taken with a Perkin Elmer UV-vis spectrometer with a slit size of $5 \mathrm{~nm}$ and range of 200-1300 nm. Dispersive Raman spectra (Kaiser Optical Systems, Inc 5000R system) are acquired with excitation wavelengths of $632.8 \mathrm{~nm}$ and $785 \mathrm{~nm}$, and Fourer Transform (FT)-Raman spectra (Raman accessory to Digilab 7000 FTS) are also acquired with a laser excitation wavelength of $1064 \mathrm{~nm}$. Baseline correction and peak fitting analysis are applied on all acquired spectra for the determination of peak positions.

\section{RESULTS AND DISCUSSION}

Figure 1 shows the Raman radial breathing mode (RBM) peaks of a spectrum from a sample of the HiPco SWNTs dispersed in an aqueous solution of NaDDBS obtained with the incident laser wavelength of $632.8 \mathrm{~nm}$. As indicated, seven resonant peaks are observed at $\omega_{R B M}=$ 197.79, 222.20, 238.53, 254.31, 265.07, 284.49, and $297.18 \mathrm{~cm}^{-1}$. The dispersive Raman data acquired with an excitation wavelength of $785 \mathrm{~nm}$ exhibit four RBM peaks at $\omega_{R B M}=172.25$, 207.62, 229.59, and $266.73 \mathrm{~cm}^{-1}$. For the FT-Raman spectra acquired with an excitation wavelength of $1064 \mathrm{~nm}$, a single RBM peak is present at $\omega_{R B M}=267.56 \mathrm{~cm}^{-1}$. The SWNT diameters, $d_{t}$, are calculated from the RBM peak positions using the expression

$$
\omega_{R B M}=239\left(\mathrm{~cm}^{-1} \mathrm{~nm}\right) / d_{t}(\mathrm{~nm})+8.5 \mathrm{~cm}^{-1}
$$




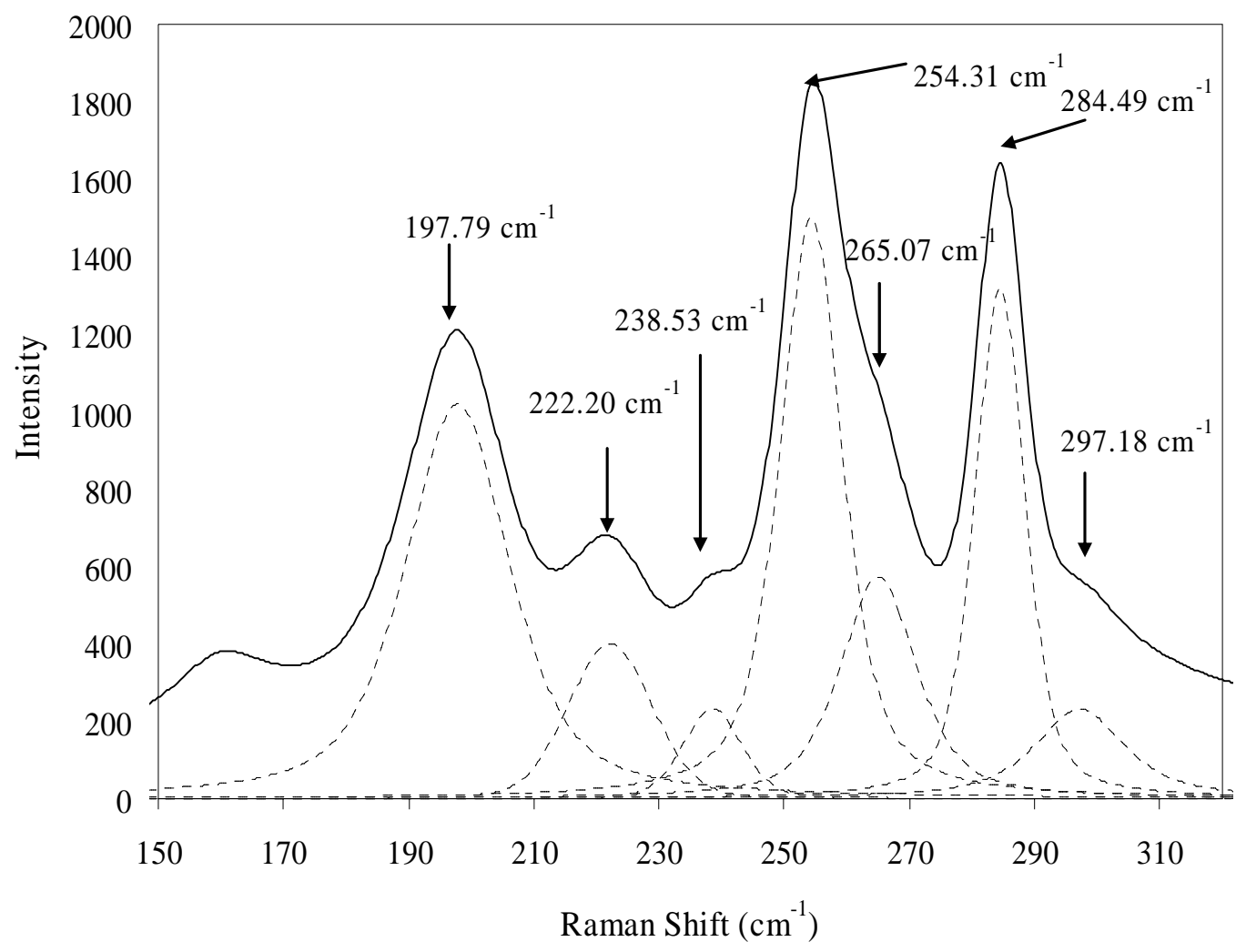

Figure 1. Radial breathing mode (RBM) Raman scattering peaks from SWNTs obtained with an incident laser excitation wavelength of $632.8 \mathrm{~nm}$. The solid trace is the acquired data, and the dotted lines indicate resulting peaks fitted through spectral analysis.

which has been found experimentally to be most accurate for isolated HiPco SWNTs [10]. A list of $(\mathrm{n}, \mathrm{m})$ pairs producing the $d_{t}$ values within $10 \%$ of each obtained from a RBM peak position is then constructed. The interband transition energy for each $(n, m)$ pair is then calculated using a first nearest neighbor tight binding energy expression [11]

$$
\begin{aligned}
E(k)= & \pm \gamma_{o}\left\{3-\cos \vec{k} \cdot \vec{a}_{1}-\cos \vec{k} \cdot \vec{a}_{2}-\cos \vec{k} \cdot\left(\vec{a}_{1}-\vec{a}_{2}\right)\right. \\
& \left.+\sqrt{3} \sin \vec{k} \cdot \vec{a}_{1}-\sqrt{3} \sin \vec{k} \cdot \vec{a}_{2}-\sqrt{3} \sin \vec{k} \cdot\left(\vec{a}_{1}-\vec{a}_{2}\right)\right\}^{1 / 2}
\end{aligned}
$$

where $\gamma_{o}=2.9 \mathrm{eV}$ is the nearest neighbor overlap energy, $\vec{k}$ is a wave vector originated from a Fermi level that is normally denoted as the K-point in a two-dimensional hexagonal reciprocal lattice, and $\vec{a}_{1}$ and $\vec{a}_{2}$ are the primitive lattice translation vectors of a graphite sheet. The actual calculation uses $\vec{k}=\vec{k}_{z}+\vec{k}_{\theta}$, where $\vec{k}_{z}$ and $\vec{k}_{\theta}$ are the components which are parallel and perpendicular to the SWNT axis, respectively. The (n, m) dependence of $E(k)$ originates from that in the expressions of the chiral angle and discrete $\vec{k}_{\theta}$ [11]. At a given $\vec{k}_{\theta}$ the magnitude of $E(k)$ is numerically calculated at $\vec{k}_{z, o}$ where $d E /\left.d k_{z}\right|_{k_{z}=k_{z, o}}=0$. To include the density of 
states (DOS) peak split effect [11,12], the calculation is repeated for $-\vec{k}_{z, o}$, and the average of the two is doubled to compute the interband transition energy. The interband transition energies thus calculated are compared with the peak positions in the optical absorption spectrum shown in Figure 2, and the results are summarized in Table I.

As shown in Table I, the chiralities present in the batch of SWNTs from which the surfactant suspensions were made include $(4,16),(6,12)$, and $(8,8)$ among the metallic nanotubes. The data also show matches for the chirality assignments of $(4,11),(1,11),(2,12),(5,9)$, and $(0,11)$ for the semiconducting SWNTs. As highlighted in Table I, all three excitation wavelengths correlate to within a few percent for the chirality assignment of $(1,11)$ based on the first and second semiconducting interband transitions and the calculated diameter.

This method and resulting data demonstrate that a significant level of (n, m) characterization is possible with limited Raman data when these are directly incorporated into the optical absorption spectrum. The batch of HiPco SWNTs studied here has a rather broad diameter distribution such that the $E_{22}^{S}$ and $E_{11}^{M}$ optical absorption peaks overlap, as can be seen in Figure 2. If the low energy limit of the optical absorption spectroscopy can be lowered, one can experimentally observe a broader range of the $E_{11}^{S}$ and $E_{22}^{S}$ absorption peaks from the same SWNTs for more extensive comparison with the theoretical transition energies. The same is true

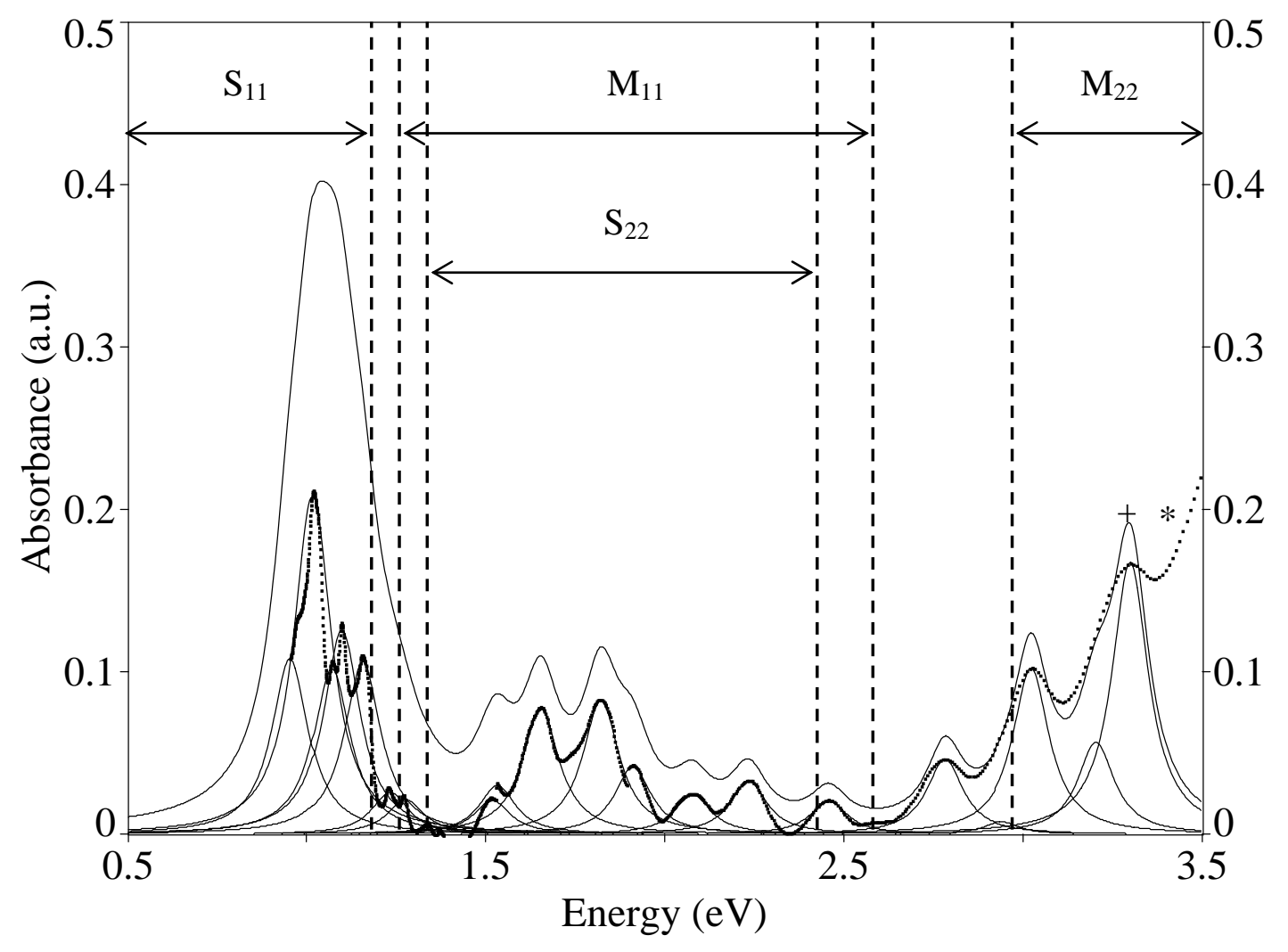

Figure 2. Optical absorption spectrum of the SWNT sample. The dotted line trace denoted by * (on the right in the figure) is the acquired, unprocessed data, and the solid line trace denoted by + is the spectrum resulting from the fitted peaks (also shown with solid line traces). The range of the interband transition energies for metallic (Mii) or semiconducting (Sii) nanotubes covered by the RBM peaks of the experimental Raman spectra are indicated by the dashed vertical lines. 
Table I. Chirality assignment of SWNTs from Raman and optical absorption data.

\begin{tabular}{|c|c|c|c|c|c|c|c|c|}
\hline Peak & $\begin{array}{l}\lambda_{\text {excit }} \\
(\mathbf{n m})\end{array}$ & $\begin{array}{l}\boldsymbol{\omega}_{\text {RBM }} \\
\left.\mathbf{( c m}^{-1}\right)\end{array}$ & $\begin{array}{l}\mathbf{d}_{\text {calculated }} \\
(\mathbf{n m})\end{array}$ & $\begin{array}{l}\mathbf{d}_{\text {theory }} \\
(\mathbf{n m})\end{array}$ & $\mathbf{( n , ~ m )}$ & Transition & $\begin{array}{l}\Delta \mathbf{E}_{\text {theory }} \\
(\mathbf{e V})\end{array}$ & $\begin{array}{l}\Delta \mathbf{E}_{\text {opt abs }} \\
(\mathbf{e V})\end{array}$ \\
\hline 1 & 785 & 172.25 & 1.4596 & 1.4528 & 4,16 & $\mathrm{M} 11$ & 1.70814 & 1.6998 \\
\hline & & & 1.4596 & 1.4528 & 4,16 & $\mathrm{M} 22$ & 3.3245 & 3.3338 \\
\hline 2 & 785 & 207.62 & 1.2003 & 1.1994 & 5,12 & $\mathrm{~S} 22$ & 1.3806 & 1.4306 \\
\hline 3 & 785 & 229.59 & 1.081 & 1.066 & 4,11 & $\mathrm{~S} 22$ & 1.5513 & 1.5522 \\
\hline 4 & 785 & 266.73 & 0.9255 & 0.9141 & 1,11 & $\mathrm{~S} 22$ & 1.8133 & 1.8368 \\
\hline & & & 0.9255 & 0.9141 & 1,11 & $\mathrm{~S} 11$ & 0.911 & 0.9368 \\
\hline 5 & 1064 & 267.56 & 0.9226 & 0.9141 & 1,11 & $\mathrm{~S} 11$ & 0.911 & 0.9368 \\
\hline & & & 0.9226 & 0.9141 & 1,11 & $\mathrm{~S} 22$ & 1.8133 & 1.8368 \\
\hline 6 & 632.8 & 265.07 & 0.9315 & 0.9141 & 1,11 & $\mathrm{~S} 22$ & 1.8133 & 1.8368 \\
\hline & & & 0.9315 & 0.9141 & 1,11 & $\mathrm{~S} 11$ & 0.911 & 0.9368 \\
\hline 7 & 632.8 & 197.79 & 1.2623 & 1.2582 & 6,12 & $\mathrm{M} 11$ & 1.9562 & 1.9104 \\
\hline 8 & 632.8 & 222.2 & 1.1184 & 1.0983 & 8,8 & $\mathrm{M} 11$ & 2.2196 & 2.2313 \\
\hline 9 & 632.8 & 238.53 & 1.039 & 1.039 & 2,12 & $\mathrm{~S} 22$ & 1.5954 & 1.6287 \\
\hline 10 & 632.8 & 254.31 & 0.9723 & 0.974 & 5,9 & $\mathrm{~S} 22$ & 1.6909 & 1.6998 \\
\hline 11 & 632.8 & 284.49 & 0.86597 & 0.8719 & 0,11 & $\mathrm{~S} 22$ & 1.9012 & 1.9104 \\
\hline & & & 0.86597 & 0.8719 & 0,11 & $\mathrm{~S} 11$ & 0.9549 & 0.9783 \\
\hline
\end{tabular}

for $E_{11}^{M}$ and $E_{22}^{M}$ if the upper limit of the optical absorption spectral range can be increased. The accuracy of the (n, $m$ ) assignment depends critically on the accuracy of the tight binding calculation. Reich et al. demonstrated that there exist practically no discrepancies among $E_{11}^{S}$ and $E_{22}^{S}$ calculated by the $a b$ initio, nearest neighbor tight binding and third neighbor tight binding methods [13]. However, the nearest neighbor tight binding method overestimates the values of $E_{11}^{M}$ by approximately $10 \%$ as compared to those computed by the other two methods, and the discrepancy is much more pronounced in the values of $E_{22}^{M}$. Thus, our current assignment of (n, m) values for metallic SWNTs may not be totally accurate, necessitating the use of the third nearest neighbor tight binding method in the future work.

\section{CONCLUSIONS}

In this paper, we demonstrate a near complete characterization of chirality of a batch of SWNTs by directly incorporating the optical absorption spectrum into limited RBM Raman spectra. The peaks of these two types of spectra almost completely match over the entire spectral range of the optical absorption spectra. It is also noted that the accuracy of chirality characterization can be enhanced both for the semiconducting and the metallic SWNTs if the optical absorption spectral range is extended. Involving only non-specialized tests of Raman and optical absorption spectroscopy and a simple analysis procedure, the method in the future can be developed as a practical tool for routine characterization of the chirality distribution of batches of SWNTs. 


\section{ACKNOWLEDGMENTS}

The authors thank Dr. Kristin Pawlowski Burney for her assistance in sample preparation. The research was performed while the author P. Williams held a National Research Council Research Associateship Award at NASA Langley Research Center and C. Mayweather was a participant in the NASA Summer Scholars Program.

\section{REFERENCES}

1. R. Saito, G. Dresselhaus, and M.S. Dresselhaus, Physical Properties of Carbon Nanotubes. (Imperial College Press, London, 2001).

2. M.J. O'Connell, S.M. Bachilo, C.B. Huffman, V.C. Moore, M.S. Strano, E.H. Haroz, K.L. Rialon, P.J. Boul, W.H. Noon, C. Kittrell, J. Ma, R.H. Hauge, R.B. Weisman, and R.E. Smalley, Science 297 (5581), 593 (2002).

3. M.S. Strano, S.K. Doorn, E.H. Haroz, C. Kittrell, R.H. Hauge, and R.E. Smalley, Nano Letters 3 (8), 1091 (2003).

4. A. Hartschuh, H.N. Pedrosa, L. Novotny, and T.D. Krauss, Science 301 (5638), 1354 (2003).

5. C. Fantini, A. Jorio, M. Souza, M.S. Strano, M.S. Dresselhaus, and M.A. Pimenta, Phys. Rev. Lett. 93, 147406 (2004).

6. H. Telg, J. Maultzsch, S. Reich, F. Hennrich, and C. Thomsen, Phys. Rev. Lett. 93 (17), 177401 (2004).

7. P. Nikolaev, M.J. Bronikowski, R.K. Bradley, F. Rohmund, D.T. Colbert, K.A. Smith, and R.E. Smalley, Chem. Phys. Lett. 313 (1-2), 91 (1999).

8. M.F. Islam, E. Rojas, D.M. Bergey, A.T. Johnson, and A.G. Yodh, Nano Letters 3 (2), 269 (2003).

9. J.I. Paredes and M. Burghard, Langmuir 20 (12), 5149 (2004).

10. A. Kukovecz, C. Kramberger, V. Georgakilas, M. Prato, and H. Kuzmany, European Physical Journal B 28 (2), 223 (2002).

11. S. Reich and C. Thomsen, Physical Review B (Condensed Matter and Materials Physics) 62 (7), 4273 (2000).

12. R. Saito, G. Dresselhaus, and M.S. Dresselhaus, Physical Review B (Condensed Matter and Materials Physics) 61 (4), 2981 (2000).

13. S. Reich, C. Thomsen, and P. Ordejon, Physical Review B (Condensed Matter and Materials Physics) 65, 155411 (2002). 